Article

\title{
A New Chaotic System with Positive Topological Entropy
}

\author{
Zhonglin Wang ${ }^{1, *}$, Jian Ma ${ }^{1,2}$, Zengqiang Chen ${ }^{2,3}$ and Qing Zhang ${ }^{3}$ \\ ${ }^{1}$ Department of Physics and Electronics, Binzhou University, Binzhou 256600, China \\ ${ }^{2}$ College of Computer and Control Engineering, Nankai University, Tianjin 300071, China; \\ E-Mail: chenzq@ nankai.edu.cn (Z.C.) \\ ${ }^{3}$ College of Science, Civil Aviation University of China, Tianjin 300300, China; \\ E-Mail: qzhang@cauc.edu.cn
}

* Author to whom correspondence should be addressed; E-Mail: wangzhonglin@bzu.edu.cn.

Academic Editors: Guanrong Chen, C.K. Michael Tse, Mustak E. Yalcin, Hai Yu and Mattia Frasca

Received: 26 April 2015 / Accepted: 29 July 2015 / Published: 3 August 2015

\begin{abstract}
This paper introduces a new simple system with a butterfly chaotic attractor. This system has rich and complex dynamics. With some typical parameters, its Lyapunov dimension is greater than other known three dimensional chaotic systems. It exhibits chaotic behavior over a large range of parameters, and the divergence of flow of this system is not a constant. The dynamics of this new system are analyzed via Lyapunov exponent spectrum, bifurcation diagrams, phase portraits and the Poincaré map. The compound structures of this new system are also analyzed. By means of topological horseshoe theory and numerical computation, the Poincaré map defined for the system is proved to be semi-conjugate to 3 -shift map, and thus the system has positive topological entropy.
\end{abstract}

Keywords: chaos; compound structures; topological horseshoe; topological entropy

\section{Introduction}

Chaos is a very interesting nonlinear phenomenon in nature, and can be applied to many fields of secure communications [1,2], nonlinear circuits [3,4], synchronization [5-7], and so on. Recently, it has been noticed that purposefully creating chaos becomes a hot issue in theory and application in nonlinear science.

Since the discovery of the famous Lorenz system in 1963 [8], a number of chaotic systems have been presented. In 1976, Rössler proposed an even simpler three-dimensional (3D) chaotic system [9]. 
In 1983, Chua constructed the notable Chua circuit which exhibits two-scroll chaotic behavior [10]. In 1994, Sprott presented nineteen distinct simple chaotic flows with either five terms and two nonlinearities or six terms and one nonlinearity [11]. In 1999, Chen and Ueta constructed a new system using an engineering feedback anti-control approach [12]. Moreover, many other discrete or continuous chaotic systems have also been proposed, such as Logistic map [13], Hénon map [14], Lozi map [15], Lü system [16].

Although so many chaotic systems have been proposed, finding a new chaotic system with a different topological structure and complex dynamical behavior is still a challenging task. Therefore, it is significant to introduce and analyze a new chaotic system before the chaos applied in engineering applications [1-7]. Based on this point, in this paper we propose a new 3D autonomous chaotic system which has the following characteristics compared with other known 3D chaotic systems:

(a) The system has a simple algebraic structure including one constant term, two linear terms and two nonlinear terms.

(b) With some typical parameters, the Lyapunov dimension of the considered system is greater than other known 3D chaotic systems

(c) The divergence of flow of the proposed system is not a constant but is always less than zero, while it's a negative constant for other known $3 \mathrm{D}$ chaotic systems. The bigger the divergence is, the more scattered the phase trajectory is.

(d) The proposed chaotic attractor has a compound structure which can be demonstrated using a half-image operation to obtain the left or the right half-image attractors.

(e) The system exhibits chaotic behavior over a large range of parameters.

Therefore, this chaotic system possesses simple algebraic structures but has complicated dynamics. It is necessary to thoroughly analyze its dynamical behaviors.

Currently, the chaotic behavior of the dynamical system is mainly confirmed by the Lyapunov exponents and the numerical simulation. However, sometimes the Lyapunov exponents are not precise enough because of the existence of numerical simulation error. Topological horseshoe theory [17] can allay the numerical simulation error. The existence of a topological horseshoe is recognized as one of the most important signatures of chaos. The topological horseshoe theory has provided a very powerful technique to study the essential horseshoe dynamics in nonlinear chaotic systems.

Many continuous or discrete chaotic systems have been confirmed to contain a horseshoe, including the Hénon map [18-20], the Lorenz system [21,22], the Rössler system [20], the fractional-order unified system [23], a class of three dimensional Glass networks [24] and a chaotic system with only one stable equilibrium [25]. However, it is still a challenging task for researchers to find a topological horseshoe in a chaotic system. For continuous systems, the topological horseshoe theory cannot be directly applied, and thus one must select a suitable Poincaré map before the application of topological horseshoe theory.

In this paper, a Poincaré section is properly chosen to obtain the Poincaré map. We prove that the Poincaré map is semi-conjugate to the 3-shift map by means of the topological horseshoe theory and numerical computation. This implies the entropy of the new system is at least $\log 3$.

The paper is organized as follows. Section 2 proposes a new simple chaotic system that does not belong to the generalized Lorenz system family, and also discusses the stability of its equilibria. 
In Section 3, the complex dynamics of the new system are further investigated by Lyapunov exponents, phase portraits, and bifurcation diagrams. Moreover, the compound structures of the new chaotic attractor are also demonstrated by using a half-image operation to obtain the left or the right half-image attractor. In Section 4, based on the topological horseshoe theory, a computer-assisted proof for the existence of a horseshoe in the new system is presented. Conclusions are given in Section 5.

\section{The New Chaotic System and Its Basic Properties}

\subsection{System Description}

We add a nonlinear feedback controller $u^{T}=\left[u_{1}, u_{2}, u_{3}\right]$ to the Lorenz system as follows:

$$
\left\{\begin{array}{l}
\dot{x}=a(y-x)+u_{1} \\
\dot{y}=c x-y-x z+u_{2} \\
\dot{z}=x y-b z+u_{3}
\end{array}\right.
$$

where $x, y$ and $z$ are state variables, and $a, b, c \in \mathbb{R}^{+}$. When the nonlinear feedback controller has the following forms:

$$
\left\{\begin{array}{l}
u_{1}=a x-2 a y+y z \\
u_{2}=y-2 c x+x z-y z \\
u_{3}=b z-x y-d+x^{2}
\end{array}\right.
$$

a new system can be obtained as:

$$
\left\{\begin{array}{l}
\dot{x}=-a y+y z \\
\dot{y}=-c x-y z \\
\dot{z}=-d+x^{2}
\end{array}\right.
$$

where $d \in \mathbb{R}^{+}$. The new system only consists of a constant term $d$, two linear terms $x$ and $y$, and two quadratic terms $y z$ and $x^{2}$, whose algebraic structure is simpler than Lorenz system [8], Rössler system [9] and Chen system [12].

\subsection{Non-generalized Lorenz System}

For the generalized Lorenz system (GLS), its canonical form [26-29] is:

$$
\dot{M}=\left(\begin{array}{cc}
A & 0 \\
0 & \lambda_{3}
\end{array}\right) M+x\left(\begin{array}{ccc}
0 & 0 & 0 \\
0 & 0 & -1 \\
0 & 1 & 0
\end{array}\right) M
$$

where $M=[x, y, z]^{T}, \lambda_{3}<0$, and $A$ is a $2 \times 2$ real matrix

$$
A=\left(\begin{array}{ll}
a_{11} & a_{12} \\
a_{21} & a_{22}
\end{array}\right)
$$

its eigenvalues $\lambda_{1}>0$ and $\lambda_{2}<0,-\lambda_{2}>\lambda_{1}>-\lambda_{3}>0$. 
However, the new system (3) can be expressed as:

$$
\dot{M}=\left(\begin{array}{cc}
A & 0 \\
0 & \tilde{\lambda}_{3}
\end{array}\right) M+x\left(\begin{array}{ccc}
0 & 0 & 0 \\
0 & 0 & 0 \\
1 & 0 & 0
\end{array}\right) M+y\left(\begin{array}{ccc}
0 & 0 & 1 \\
0 & 0 & -1 \\
0 & 0 & 0
\end{array}\right) M+\left(\begin{array}{c}
0 \\
0 \\
-d
\end{array}\right)
$$

where $\tilde{\lambda}_{3}=0$ and

$$
A=\left(\begin{array}{ll}
a_{11} & a_{12} \\
a_{21} & a_{22}
\end{array}\right)=\left(\begin{array}{cc}
0 & -a \\
-c & 0
\end{array}\right)
$$

its eigenvalues $\lambda_{1}>0$ and $-\lambda_{2}=\lambda_{1}=\sqrt{a c}>\tilde{\lambda}_{3}=0$. In comparison with the existing GLS, such a result does not follow the conventional conditions $\left(-\lambda_{2}>\lambda_{1}>-\lambda_{3}>0\right)$ of the GLS, so the system (3) does not belong to the GLS.

\subsection{Equilibria}

To find the equilibria of system (3), let

$$
\left\{\begin{array}{l}
-a y+y z=0 \\
-c x-y z=0 \\
-d+x^{2}=0
\end{array}\right.
$$

and we can obtain two equilibria defined by

$$
E^{ \pm}=\left( \pm \sqrt{d}, \mp \frac{c \sqrt{d}}{a}, a\right)
$$

Proposition 1. The equilibria $E^{+}$and $E^{-}$are non-hyperbolic.

Proof. The Jacobian matrix of system (3) is given by

$$
J=\left(\begin{array}{ccc}
0 & z-a & y \\
-c & -z & -y \\
2 x & 0 & 0
\end{array}\right)
$$

For $E^{+}$and $E^{-}$, the characteristic equation of $J$ is:

$$
f(\lambda)=|\lambda I-J|=\lambda^{3}+a \lambda^{2}+2 c d \lambda / a+2 c d
$$

The eigenvalues corresponding to the equilibria $E^{+}$and $E^{-}$are:

$$
\lambda_{1}=-a, \lambda_{2}=\sqrt{-2 c d / a}, \lambda_{3}=-\sqrt{-2 c d / a}
$$

The parameters $a, c$ and $d$ are all greater than zero. $\quad \lambda_{1}$ is a negative real eigenvalue. $\lambda_{2}$ and $\lambda_{3}$ are a conjugate pair purely imaginary eigenvalues. Note that an equilibrium is said to be hyperbolic equilibrium if all eigenvalues of the Jacobian matrix have nonzero real parts. The real parts of $\lambda_{2}$ and $\lambda_{3}$ are zero, hence, the equilibria $E^{+}$and $E^{-}$are non-hyperbolic. Thus, this proof is completed. 
Since system (3) only has two non-hyperbolic equilibria, the conventional Ši'lnikov homoclinic criterion $[30,31]$ is not applicable for the system.

Remark 1. There are mainly two differences between the system (3) and the classic Lorenz system:

(a) The system (3) has only two nonzero equilibria when $a>0, c>0$ and $d>0$, while the Lorenz system has two nonzero equilibria and one zero equilibrium. Here the nonzero equilibria refer to the equilibria that are not at the origin.

(b) A nonzero constant term appears in the system (3), while it does not appear in the Lorenz system. System (3) does not belong to the existing GLS, whereas the Lorenz system is a special case of the GLS [26,27].

\section{Observation and Analysis of the New System}

As $a$ and $c$ varying, the dynamical behaviors of system (3) are further investigated by Lyapunov spectrum, bifurcation diagram, phase portrait, and so on. In the following discussion, the initial condition is fixed to $(0.1,0.1,0.1)$, and the system (3) is solved by the fourth-order Runge-Kutta method in MATLAB with the time step of 0.001. The three Lyapunov exponents of system (3) are denoted by $L_{1}, L_{2}, L_{3}$, and $L_{1}>L_{2}>L_{3}$.

\subsection{Fixing $c=6, d=80$ and Varying $a$}

The Lyapunov exponents spectrum of the system (3) with respect to parameter $a$ is shown in Figure 1. The corresponding bifurcation diagram of state variable $z$ is given in Figure 2. It is clear that the bifurcation diagram well coincides with the Lyapunov exponents spectrum. The stability of the system (3) is summarized as follows:

(1) When $a \in[1,1.25) \cup[3.54,3.55) \cup[3.84,3.86) \cup[3.94,3.98) \cup[4.18,4.19) \cup[4.58,4.91) \cup[4.99,6]$, we can obtain $L_{1}=0$, and both $L_{2}$ and $L_{3}$ are less than 0 , and the trajectories started from different initial conditions approach a closed orbit surrounding two equilibria. For example, the periodic orbits for $a=3.54,4.18,4.7$ and 4.99 are depicted in Figure 3c,e,h, respectively. When $a \in[4.6,4.85]$, there is a vertical white region, that's because the system (3) has a 2-periodic orbit within this range. The low density of trajectories leads to the low density of the points in the bifurcation diagram.

(2) When $a$ belongs to $[1.25,3.54) \cup[3.55,3.84) \cup[3.86,3.94) \cup[3.98,4.18) \cup[4.19,4.58) \cup$ $[4.91,4.99), L_{1}>0, L_{2}=0$ and $L_{3}<0$, and strange chaotic attractors will appear. When $a=1.25,3,3.98,4.19$ and 4.91, some double-scroll chaotic attractors are shown in Figure 3a,b,d,f,g, respectively. 


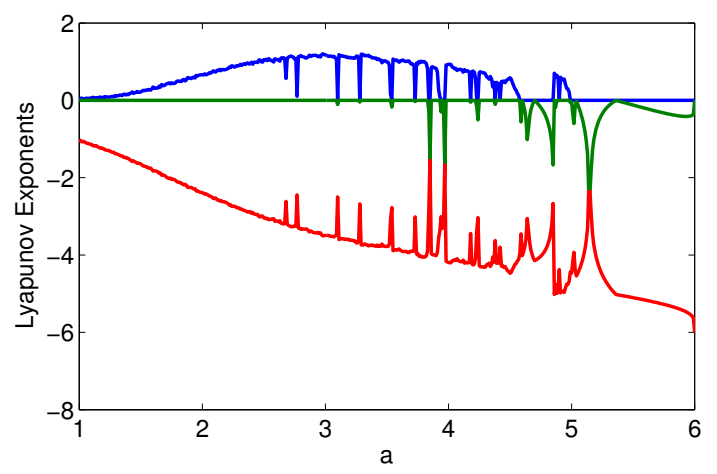

Figure 1. The Lyapunov exponent spectrum for $1 \leq a \leq 6$ with $c=6, d=80$.

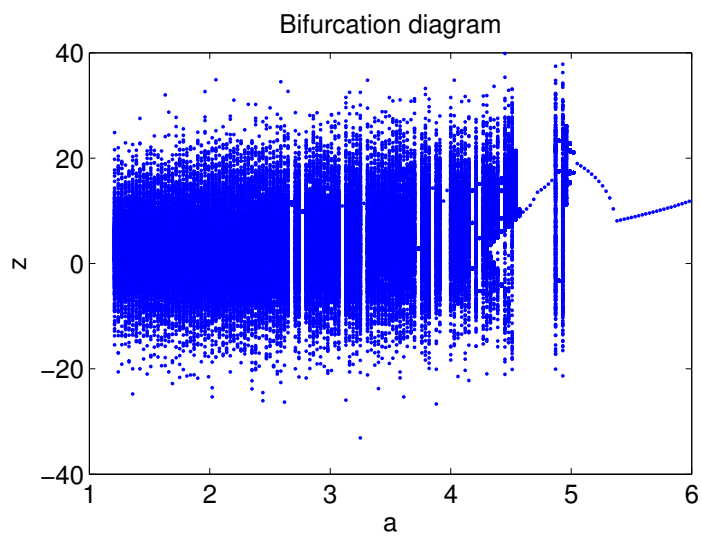

Figure 2. The Bifurcation diagram for $1 \leq a \leq 6$ with $c=6, d=80$.

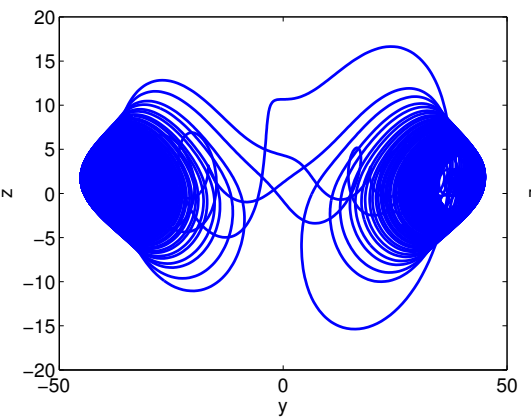

(a)

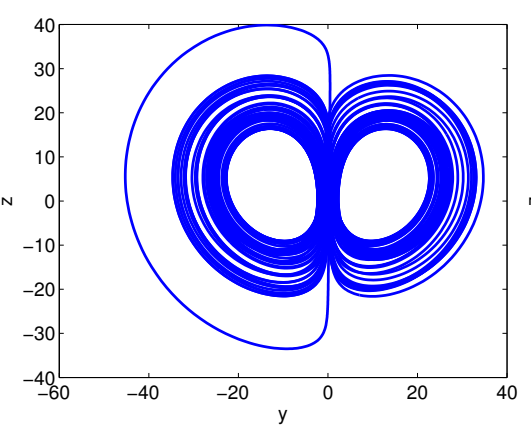

(d)

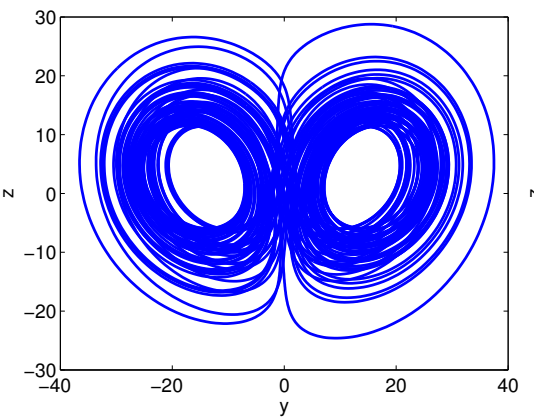

(b)

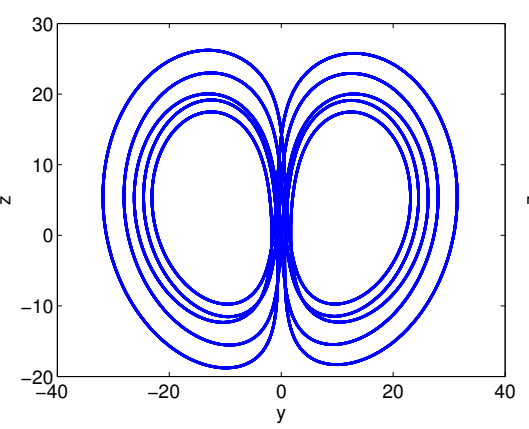

(e)

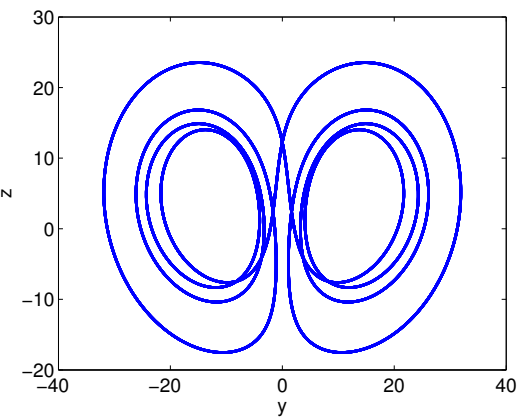

(c)

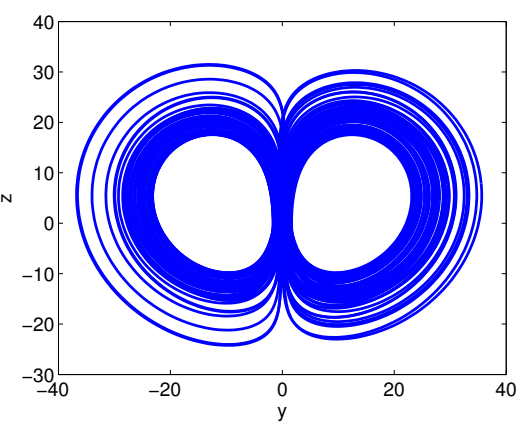

(f)

Figure 3. Cont. 


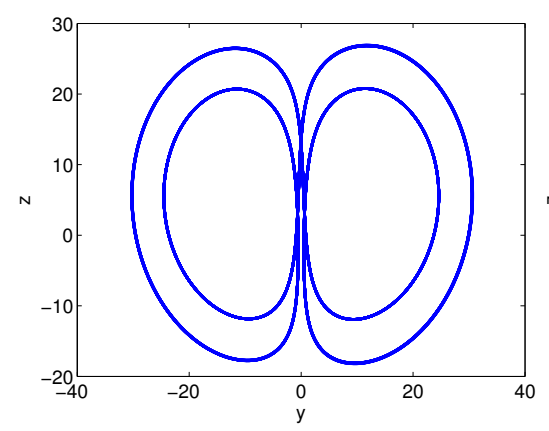

$(\mathbf{g})$

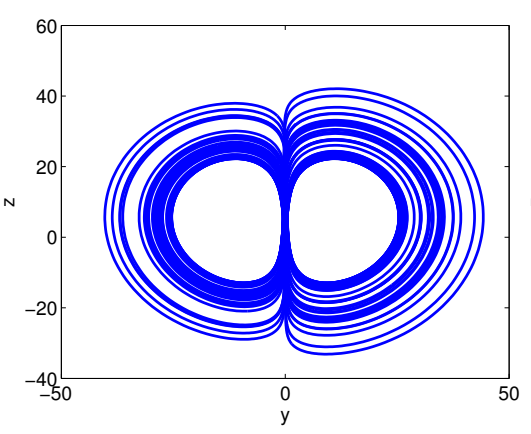

(h)

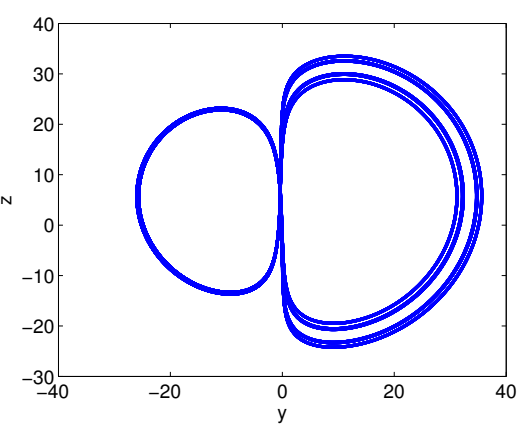

(i)

Figure 3. Phase portraits of the system (3) projected on $y-z$ plane for different $a$. (a) $a=1.25 ;(\mathbf{b}) a=3 ;$ (c) $a=3.54 ;$ (d) $a=3.98 ;(\mathbf{e}) a=4.18 ;$ (f) $a=4.19 ;$ (g) $a=4.7$; (h) $a=4.91$; (I) $a=4.99$.

\subsection{Fixing $a=3, c=6$ and Varying $d$}

Figure 4 shows the Lyapunov exponent spectrum of system (3) with respect to parameter $d$. Obviously, system (3) is chaotic for a very wide range of $d$. For instance for $d=15$, the 3D motion trajectory and its projection on $x-y, x-z$ and $y-z$ planes are demonstrated in Figure 5a-d, respectively.

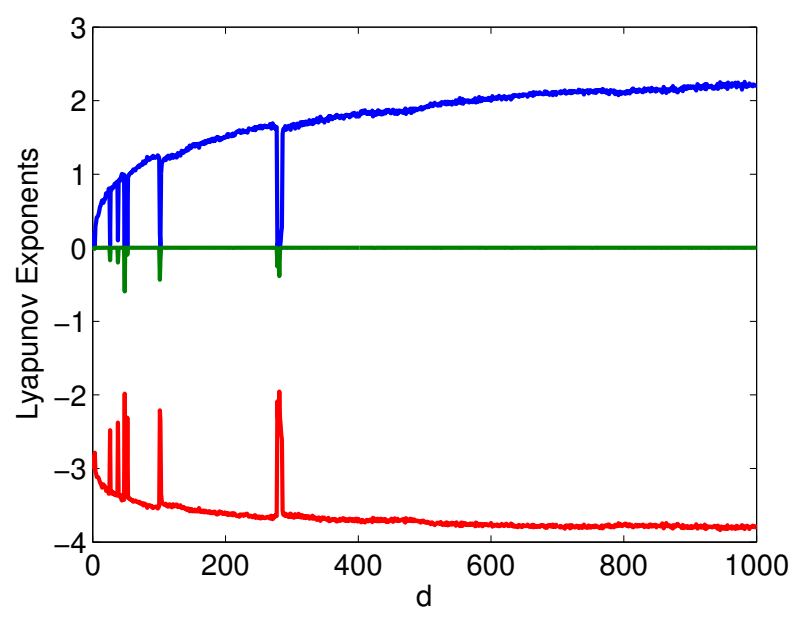

Figure 4. The Lyapunov exponent spectrum of system (3) versus the parameter $d \in[0,1000]$ with $a=3, c=6$.

Moreover, based on the Lyapunov spectrum, it is possible to obtain the Lyapunov dimension which is defined as

$$
D_{L}=j+\frac{1}{\left|L_{j+1}\right|} \sum_{i=1}^{j} L_{i}
$$

where $j$ is the maximum integer satisfying $\sum_{i=1}^{j} L_{i} \geq 0$ and $\sum_{i=1}^{j+1} L_{i}<0$. Lyapunov dimension is a measure of the geometric scaling properties or "complexity" of chaotic attractor [32]. For our chaotic system, $L_{1}>0, L_{2}=0, L_{3}<0$ and $\left|L_{1}\right|<\left|L_{3}\right|$, hence, $j=2$ and $\frac{1}{\left|L_{j+1}\right|} \sum_{i=1}^{j} L_{i}=\frac{L_{1}}{\left|L_{3}\right|}$. Substituting it into formula (12), it can be concluded that the Lyapunov dimension of the chaotic attractor is greater than 
2 and less than 3 . The fractal nature of an attractor does not only mean that this system has non-periodic orbits but also the trajectories from different conditions are in a state of separation.

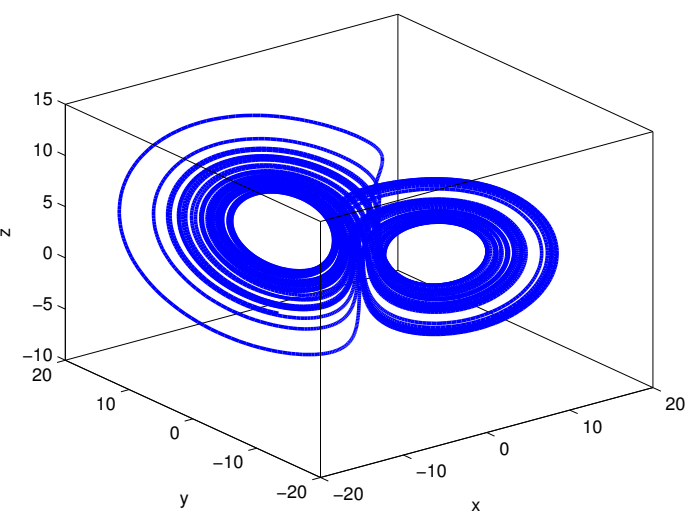

(a)

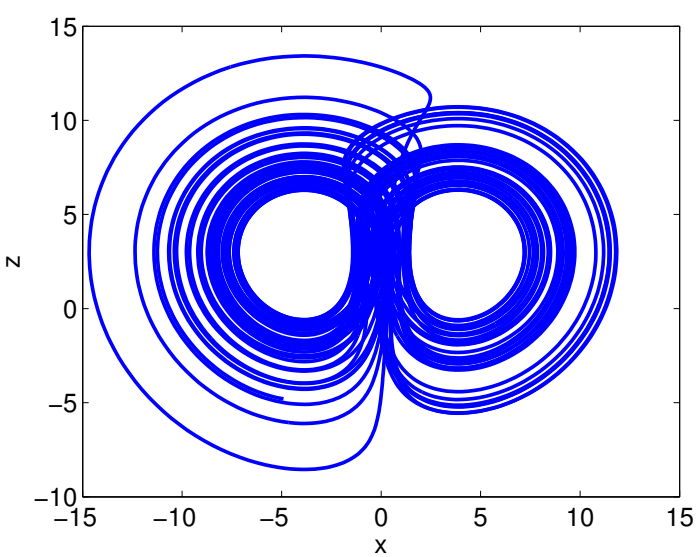

(c)

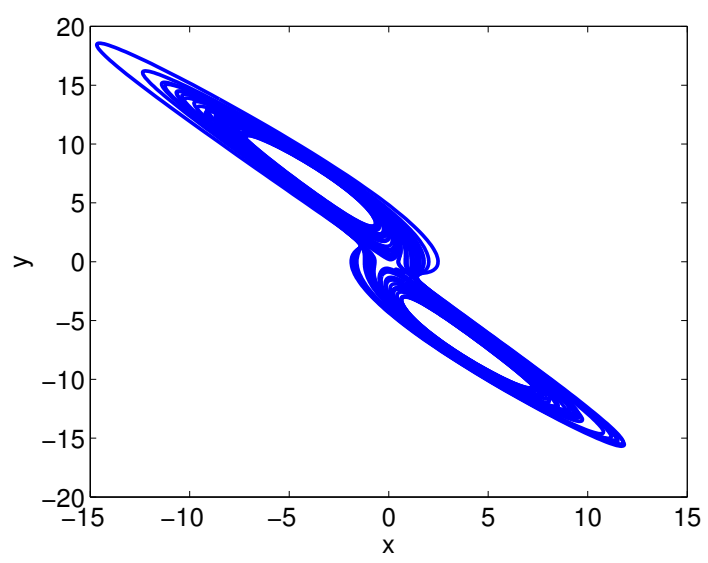

(b)

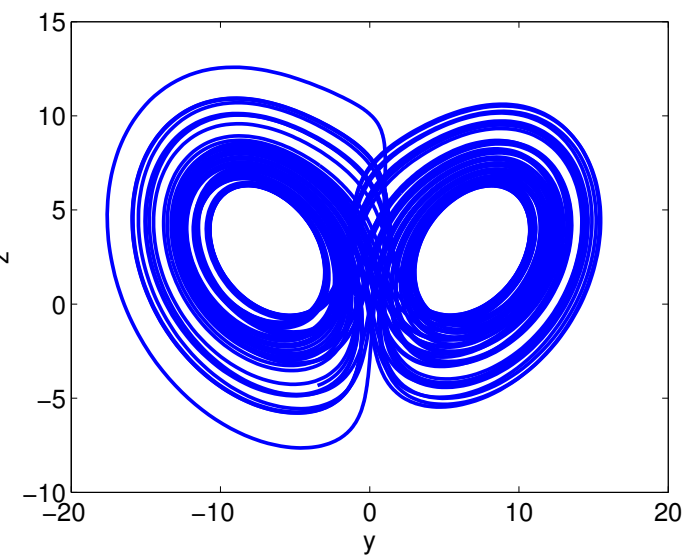

(d)

Figure 5. Phase portraits with $a=3, c=6, d=15$ (a) 3D view; (b) Projection on $x-y$ plane; (c) Projection on $x-z$ plane; (d) Projection on $y-z$ plane.

Varying $d$, the change of Lyapunov dimension of system (3) is shown in Figure 6. It is easy to see that the Lyapunov dimension continues to grow and can almost reach 2.6 as the parameter $d$ increases from 0 to 1000. The Lyapunov dimension of the Lorenz system is 2.062, the Lyapunov dimension of the Chen system is 2.168 and the Lyapunov dimensions of the Sprott B-Sprott S system are less than 2.5. Thus, there exist $a, c$ and $d$ (e.g., $a=3, c=6, d=1000$, such that the Lyapunov dimension of the system (3) is greater than other 3D known chaotic systems, such as the Lorenz system, the Chen system and Sprott B-Sprott S system.

Generally speaking, for a system, the larger the Lyapunov dimension is, the more complicated the dynamics are. Therefore, system (3) is more complex than Lorenz system, Chen system and Sprott B-Sprott S system with some typical parameters.

We study the Poincaré maps to describe bifurcation and folding properties of the proposed system. Since the plane $\Pi=\left\{(x, y, z) \in \mathrm{R}^{3} \mid x=0\right\}$ could intersect with all trajectories of system (3), we took the plane $\Pi$ as the cross-section. The Poincaré maps of the system (3) with different $d$ are shown in 
Figure $7 \mathrm{a}-\mathrm{d}$, respectively. It is easy to see that Poincaré map becomes more cluttered with the increasing of $d$. This phenomenon can be explained by the changes of Lyapunov dimension $D_{L}$ showed in Figure 6 .

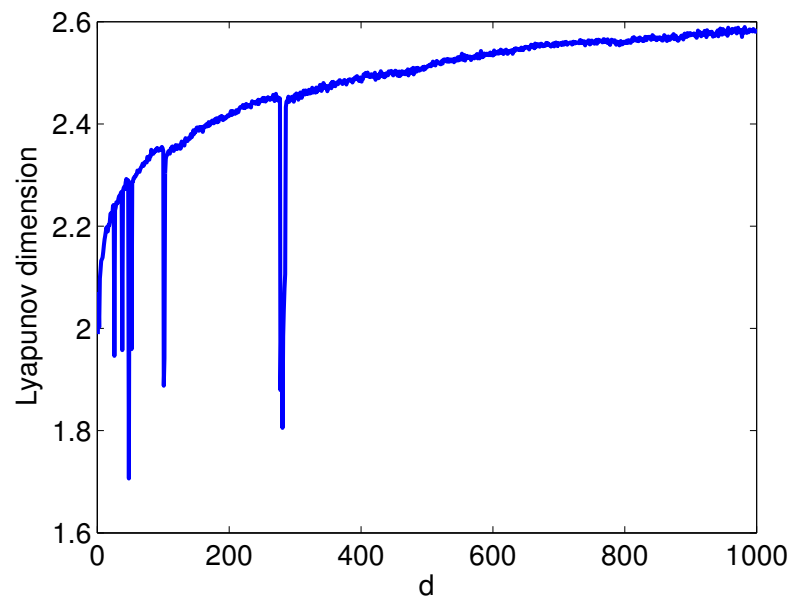

Figure 6. The changes of Lyapunov dimension versus $d$ with $a=3, c=6$.

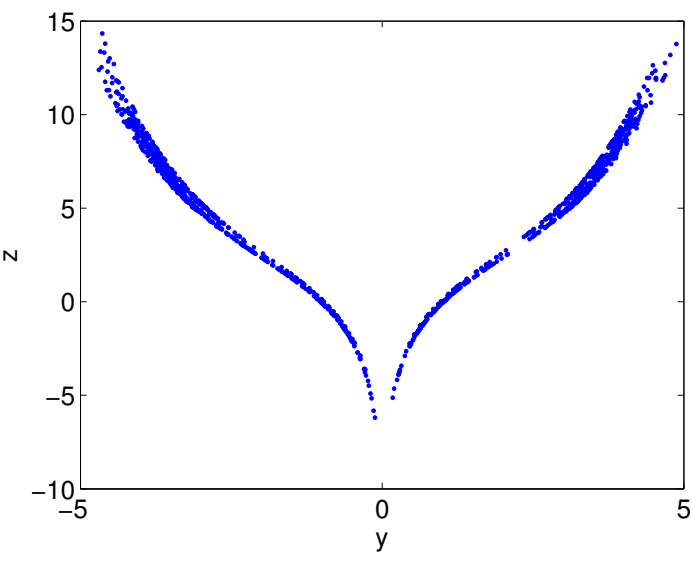

(a)

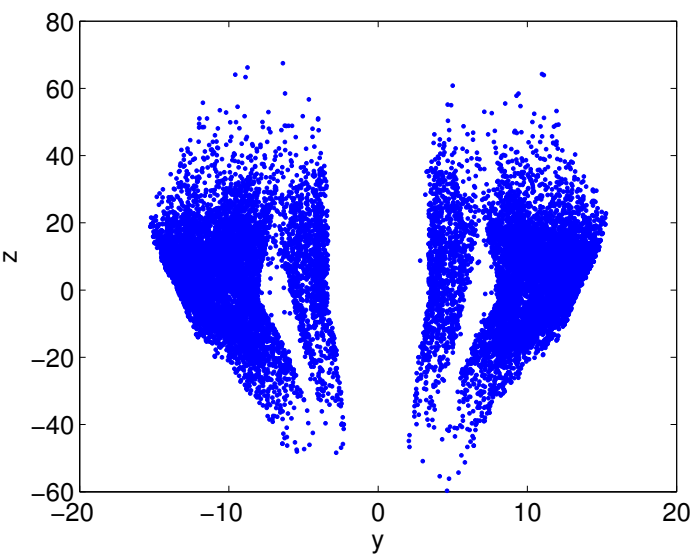

(c)

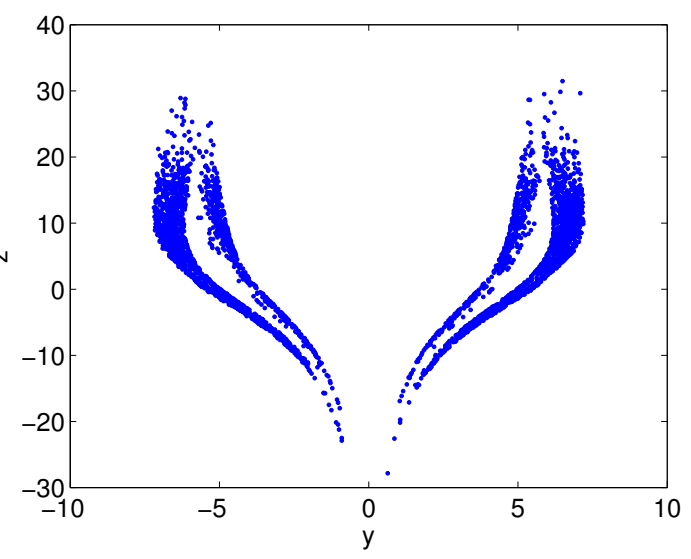

(b)

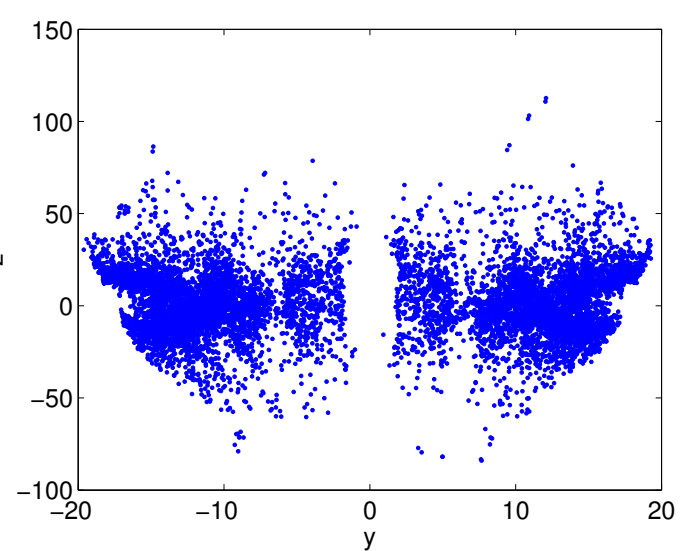

(d)

Figure 7. Poincaré map projected on $y-z$ plane for different $d$. (a) $d=15$ (b) $d=80$ (c) $d=500$ (d) $d=1000$. 


\subsection{A Dissipative System}

The divergence of the flow of the dynamical system (3) is

$$
\nabla V=\frac{\partial \dot{x}}{\partial x}+\frac{\partial \dot{y}}{\partial y}+\frac{\partial \dot{z}}{\partial z}=-z
$$

which is not a real constant value but is closely related to the state variable $z$. We cannot confirm directly that $\nabla V$ is positive or negative. Since $\nabla V=\frac{\partial \dot{x}}{\partial x}+\frac{\partial \dot{y}}{\partial y}+\frac{\partial \dot{z}}{\partial z}=L_{1}+L_{2}+L_{3}$, we investigate its Lyapunov exponents to show $\nabla V$. As shown in Figure 8, when $a$ or $d$ varies, $\nabla V$ is always less than zero, and hence system (3) is a dissipative system with an exponential rate of contraction as

$$
\frac{d V}{d t}=e^{\nabla V}
$$

It means

$$
V(t)=V_{0} e^{(\nabla V) t}
$$

Any initial volume $V_{0}$ containing the system trajectories shrinks to zero as $t \rightarrow+\infty$ at a changing exponential rate of $\nabla V$. The bigger the $\nabla V$ is, the smaller the rate of volume shrinkage is.

It is easy to see from Figure $8 \mathrm{~b}$ that $\nabla V$ continues to grow as $d$ increases from 0 to 1000 , which implies the rate of volume shrinkage decreases. Correspondingly, as shown in Figure 7, the points in Poincaré map become more cluttered as $d$ increases, which means the phase trajectory becomes more scattered. For example, when fix $a=3, c=6$, the phase trajectory for $d=80$, as shown in Figure $3 \mathrm{~b}$, is more scattered than phase trajectory for $d=15$, as shown in Figure 5.

Similarly, as shown in Figure $8 \mathrm{a}, \nabla V$ continues to decrease as $a$ increases from 1 to 6 , and hence the rate of volume shrinkage increases. Correspondingly, as shown in Figure 3, the phase trajectory becomes more concentrated as $a$ increases.

It can be concluded from above discussion that the bigger the $\nabla V$ is, the more scattered the phase trajectory is. Moreover, since differential Equation (3) is a dissipative system, system orbits are ultimately confined into a specific limit set of zero volume, and the asymptotic motion settles onto an attractor. Therefore, the existence of attractor is proved.

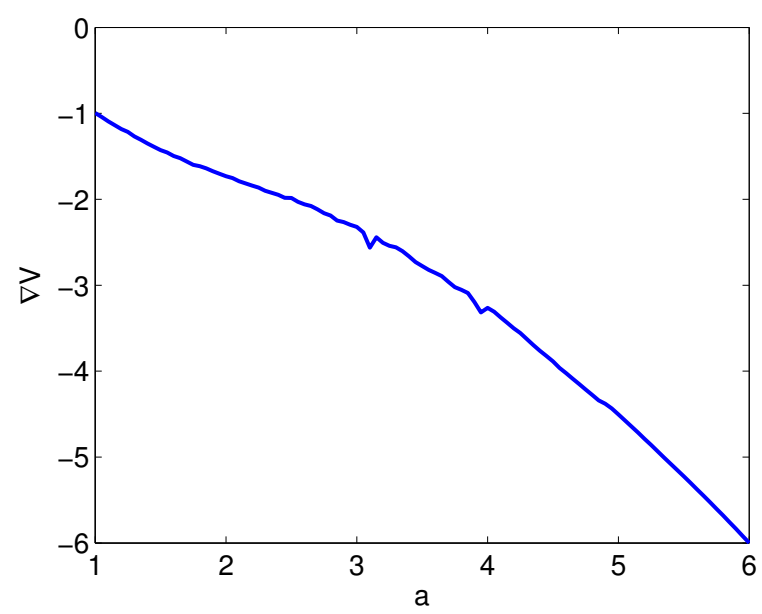

(a)

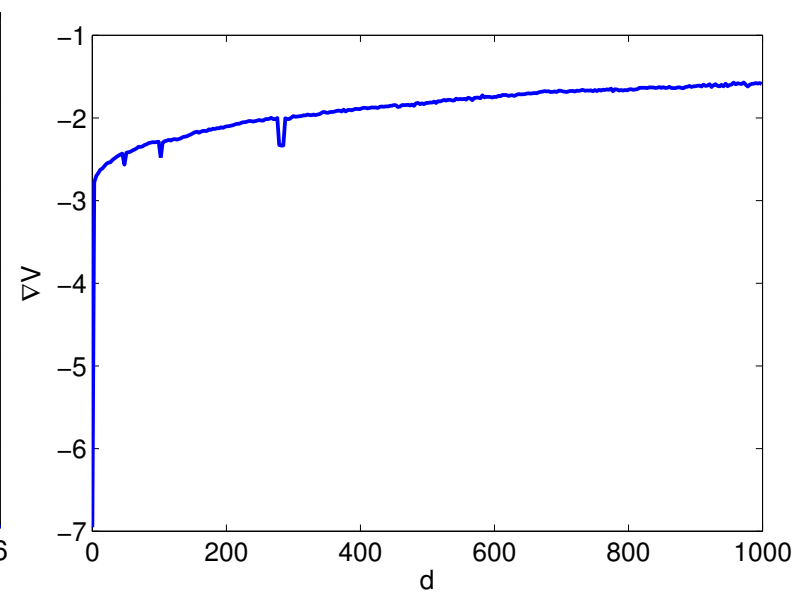

(b)

Figure 8. (a) The change of $\nabla V$ versus $a$ with $c=6, d=80$; (b) The change of $\nabla V$ versus $d$ with $a=3, c=6$; 


\subsection{Compound Structures}

From Figures 3 and 5, it can be seen that the system (3) has a double-scroll butterfly chaotic attractor. Compound structures of an attractor can be obtained by merging together two simple attractors after performing a half-image operation to obtain the left or the right half-image attractors [33]. Such an operation can be revealed by adding a linear term $k z$ into the second equation of the system (3) as follows:

$$
\left\{\begin{array}{l}
\dot{x}=-a y+y z \\
\dot{y}=-c x-y z+k z \\
\dot{z}=-d+x^{2}
\end{array}\right.
$$

where $k$ is a control parameter.

If we let $a=3, c=6, d=15$, the left or the right half-image attractors of the original attractor (Figure 5) at specific values of the parameter $k$ are shown in Figure 9. When $k=1.95$, a left half-image of the original attractor (Figure 5d) can be isolated, as shown in Figure 9a. In contrast, when $k=-1.95$, another right half-image of the original attractor (Figure $5 \mathrm{~d}$ ) can be isolated, as shown in Figure $9 \mathrm{~b}$. In other words, Figure 5d is separated into Figure 9a,b. Both of the left and right single-scroll can be merged together as a compound structure in Figure 9c. If let $k=0$, the system (14) degenerates into the system (3) and a double-scroll attractor appears in the system (14) as shown in Figure 9d.

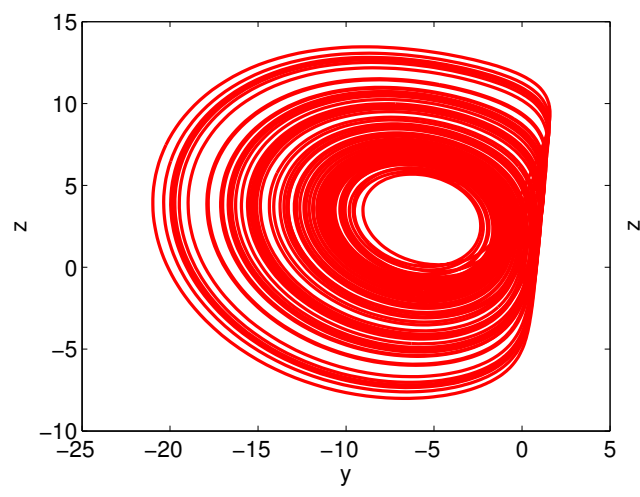

(a)

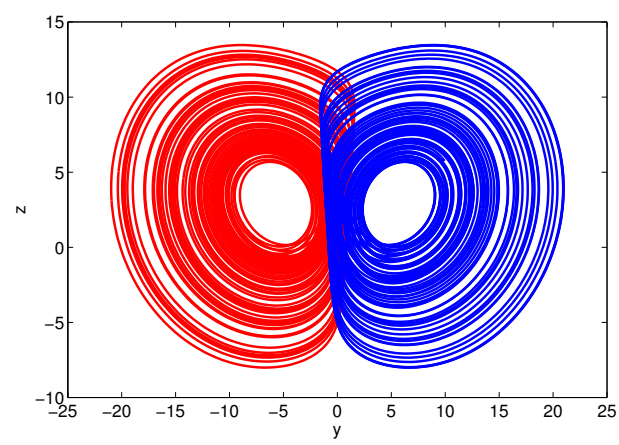

(c)

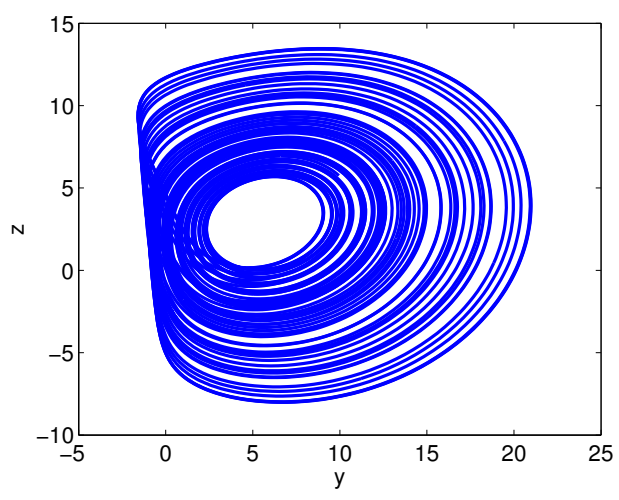

(b)

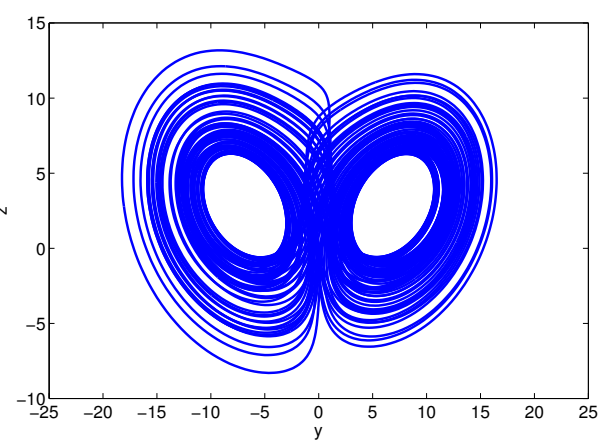

(d)

Figure 9. (a) A left half-image chaotic attractor for the $y-z$ plane at $k=1.95$; (b) A right half-image chaotic attractor for the $y-z$ plane at $k=-1.95$; (c) A superposition of (a) and (b); (d) A chaotic attractor for $y-z$ plane at $k=0$. 
When $k \in[-2.5,2.5]$, the bifurcation diagram of state variable $y$ is shown in Figure 10 . Figure 11 demonstrates gradual development of forming mechanisms of the compound structures. Different dynamical behaviors of system (14) can be summarized as follows:

(1) When $|k| \geq 2.26$, the system (14) has limit cycles. For example, Figure 11a shows a limit cycle at $k=2.4$.

(2) When $2.1<|k|<2.26$, there are period-doubling bifurcations. For example, Figure 11b,c show such period-doubling bifurcations at $k=2.2$ and 2.12, respectively.

(3) When $1.92 \leq|k| \leq 2.1$, the system (14) becomes a left (or a right) half-image attractor as shown in Figure 9a,b.

(4) When $0.5 \leq|k|<1.92$, the system demonstrates partial attractors, which are bounded. For example, Figure 11d shows a partially-right, dominantly-left, attractor at $k=0.9$.

(5) For $|k|<0.5$, the system exhibits a complete attractor. For example, Figure 5 shows the complete attractor at $k=0$.

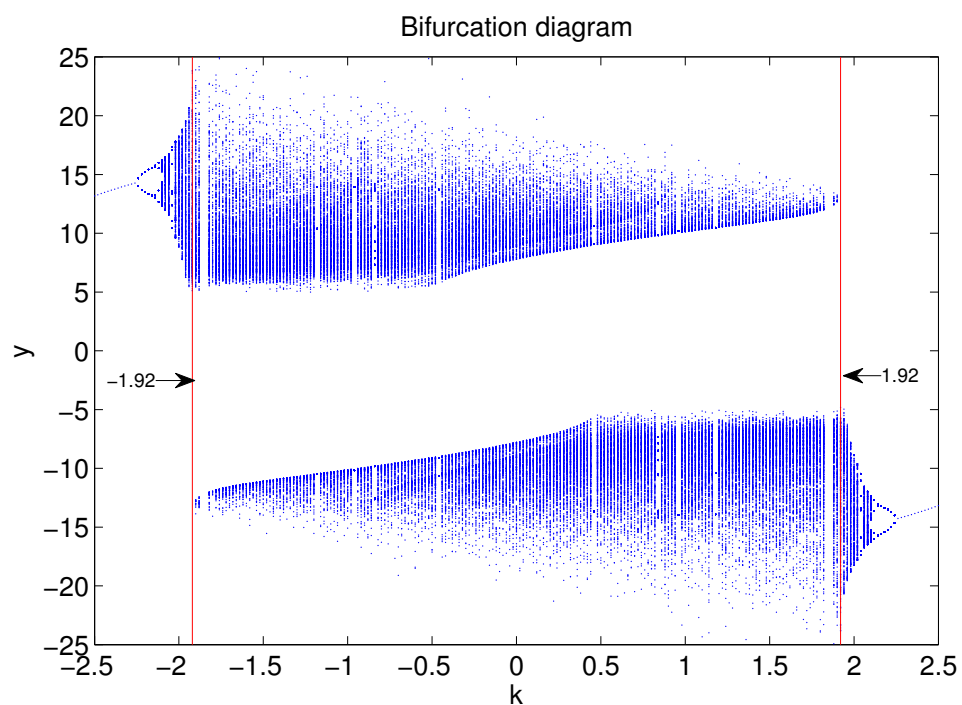

Figure 10. Bifurcation diagram of the system (14) for $-2.5<k<2.5$ with $a=3, c=6, d=15$.

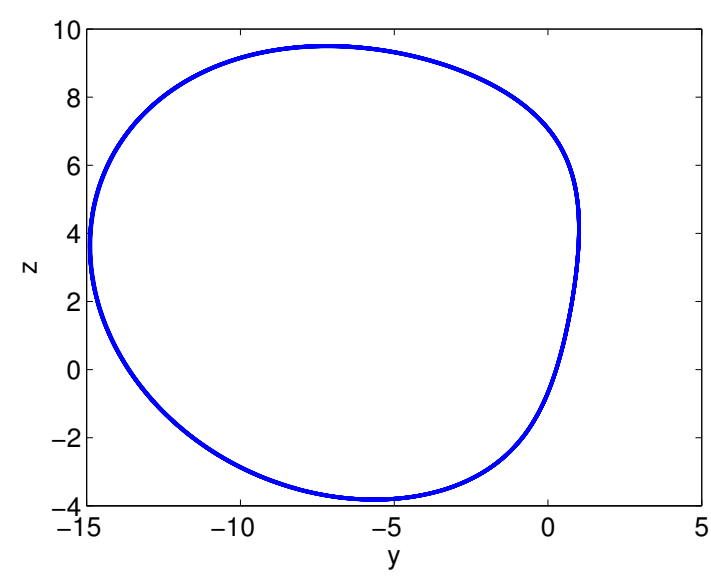

(a)

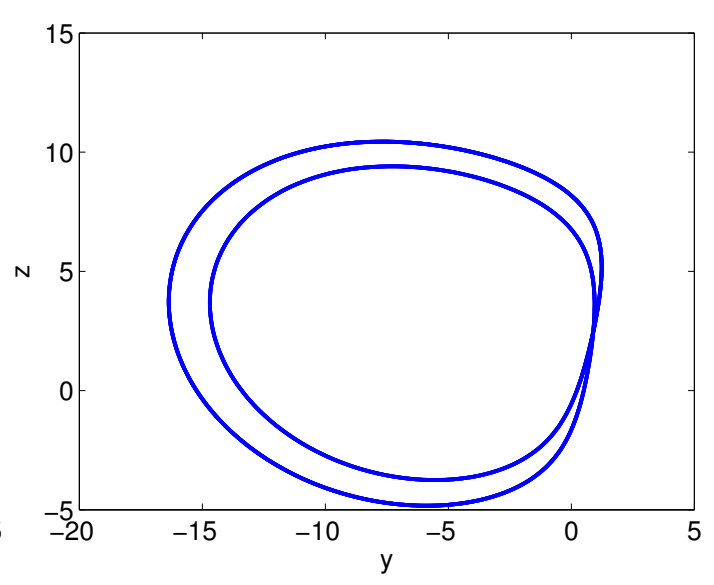

(b)

Figure 11. Cont. 


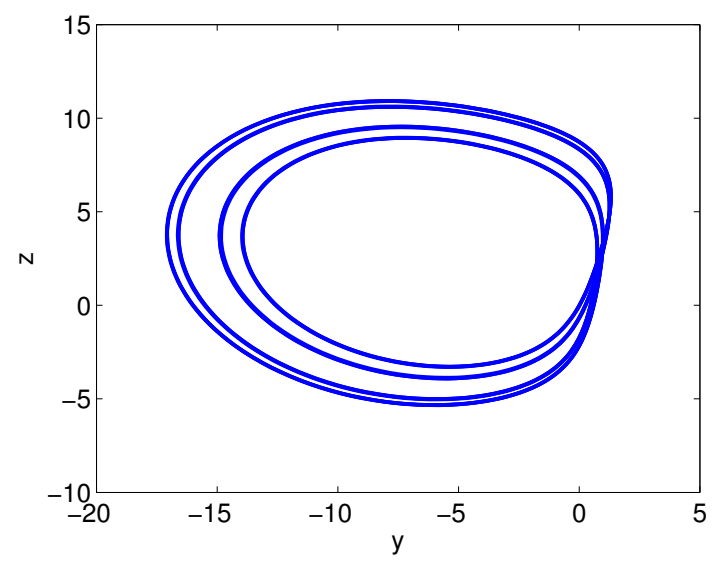

(c)

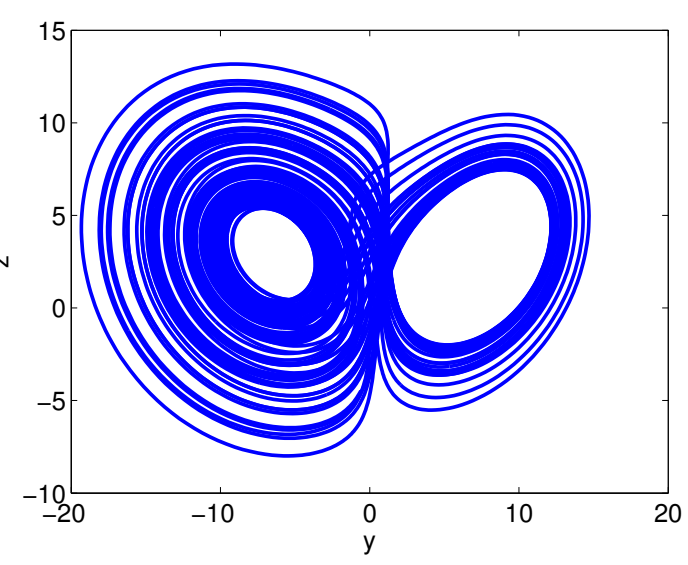

(d)

Figure 11. Phase portraits of the system (14) at (a) $k=2.4$, (b) $k=2.2$, (c) $k=2.12$, (d) $k=0.9$.

According to Figures 9 and 11, one can see that the new attractor has a compound structure, composed of two simple attractors and each emerges from some simple limit cycle (see Figure 11a).

\section{Topological Horseshoe Analysis for the New Chaotic System}

Based on the topological horseshoe theory [34,35], a rigorous computer-assisted proof for the existence of a horseshoe in the new chaotic system is presented in this section.

\subsection{Review of a Topological Horseshoe Theory}

In this subsection, we review some basic aspects about the construction and properties of the standard topological horseshoe.

Let $S_{m}=\{0,1,2, \ldots, m-1\}$ be the set of nonnegative successive integers from 0 to $m-1$, and $\sum_{m}$ be the collection of all bi-infinite sequences with their elements of $S_{m}$, i.e., $s \in \sum_{m}$ implies $s=\left\{\ldots, s_{-n}, \ldots s_{-1}, s_{0}, s_{1}, \ldots, s_{n}, \ldots\right\}, s_{i} \in S_{m}, \forall i$. If we consider another sequence $\bar{s}=\left\{\ldots, \bar{s}_{-n}, \ldots \bar{s}_{-1}, \bar{s}_{0}, \bar{s}_{1}, \ldots, \bar{s}_{n}, \ldots\right\}, \bar{s}_{i} \in S_{m}, \forall i$, then, the distance of between $s$ and $\bar{s}$ can be defined as follows:

$$
d(s, \bar{s})=\sum_{-\infty}^{\infty} \frac{1}{2^{|i|}} \frac{\left|s_{i}-\bar{s}_{i}\right|}{1+\left|s_{i}-\bar{s}\right|}
$$

With the distance defined as above, $\sum_{m}$ is a metric space, and it is well known that $\sum_{m}$ is compact, totally disconnected and perfect [36], that is a Cantor set.

Now define an m-shift map $\sigma: \sum_{m} \rightarrow \sum_{m}$ as $\sigma(s)_{i}=s_{i+1}$, and then put Proposition 2 as:

Proposition 2. [36] The shift map $\sigma$ has the following properties:

(i) $\sigma\left(\sum_{m}\right)=\sum_{m}$;

(ii) $\sigma$ is continuous;

(iii) $\sigma$ has countable many periodic orbits;

(iv) $\sigma$ has uncountable many aperiodic orbits;

(v) $\sigma$ has a dense orbit. 
Note that the dynamics of $\sigma$ on $\sum_{m}$ display sensitive dependence on initial conditions on a closed invariant set, and therefore is chaotic. (See $[36,37]$ for proofs of the above statements.)

Let $X$ be a metric space, $D$ be a compact subset of $X$, and $f: D \rightarrow X$ be such a map that there exist $m$ mutually disjoint subset $D_{1} \ldots, D_{m-1}$ and $D_{m}$ of $D$. The restriction of $f$ to each $D_{i}$, i.e., $f \mid D_{i}$ is continuous.

Definition 1. [38] Let $\gamma$ be a compact subset of $D$, such that for each $1 \leq i \leq m, \gamma_{i}=\gamma \cap D_{i}$ is nonempty and compact, then $\gamma$ is called a connection with respect to $D_{1} \ldots, D_{m-1}$ and $D_{m}$.

Let $F$ be a family of connections with respect to $D_{1} \ldots, D_{m-1}$ and $D_{m}$ satisfying the following property: $\gamma \in F \Rightarrow f\left(\gamma_{i}\right) \in F$ for every $i \in\{1, \ldots m\}$. Then $F$ is said to be a f-connected family with respect to $D_{1} \ldots, D_{m-1}$ and $D_{m}$.

Theorem 1. [39] Suppose that there exists a f-connected family $F$ with respect to $D_{1} \ldots, D_{m-1}$ and $D_{m}$. Then there exists a compact invariant set $K \subset D$, such that $f \mid K$ is semi-conjugate to $m$-shift.

Theorem 2. [37] Let $X$ be a compact metric space, and $f: X \rightarrow X$ a continuous map. If there exists an invariant set $\Lambda \subset X$ such that $f \mid \Lambda$ is semi-conjugate to the $m$-shift $\sigma$, then ent $(f) \geq \operatorname{ent}(\sigma)=\log m$ where ent $(f)$ denotes the entropy of the map $f$.

For the concept of topological entropy, we address the reader to [37]. Positivity of the entropy is one of the most commonly used definitions of chaos.

\subsection{Horseshoe in the Poincaré Map for the Proposed System}

In this subsection, we let $a=3, c=6$ and $d=15$. In order to apply the topological horseshoe theory to a continuous system, we should find a proper Poincaré map. First, a cross-section $P$ is selected on the plane $\Gamma=\left\{(x, y, z) \in \mathrm{R}^{3} \mid \mathrm{y}=0\right\}$, as shown in Figure 12. The four vertices of the cross-section $P$ are $(0,0,-10),(3.5,0,-10),(0,0,20)$ and $(3.5,0,20)$.

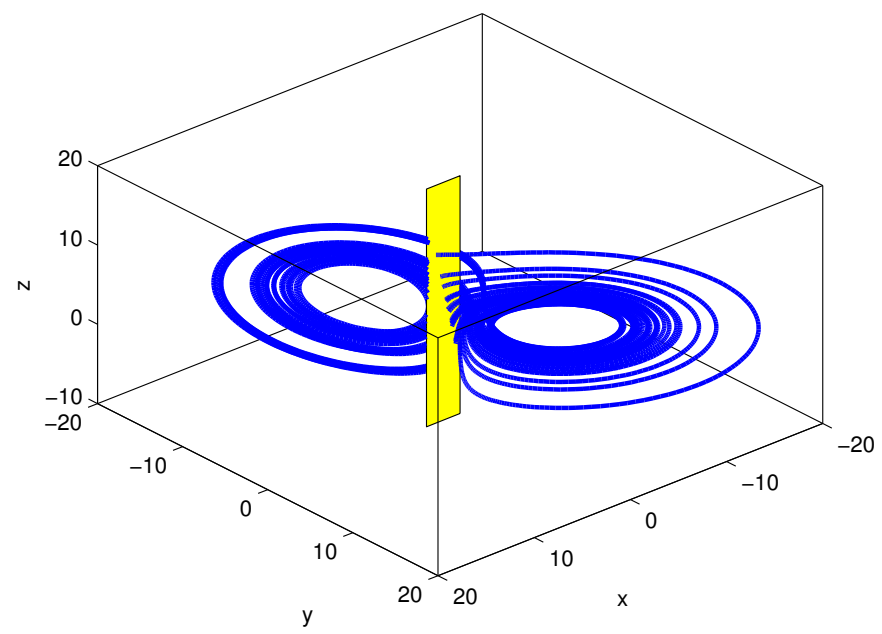

Figure 12. The attractor of system (3) and a suitable cross section.

Then, the corresponding Poincaré map $H: P \rightarrow P$ is defined as follows: for each $p \in P$, $H(p)$ is the first return intersection point with $P$ under the flow of system (3) with the initial 
condition $p$. The determination of the cross-section $P$ and the iteration number of Poincare map $H$ need multiple attempts.

We found three mutually disjoint quadrilaterals $D_{1}, D_{2}$ and $D_{3}$ in $P$.

The four vertices of $D_{1}$ are as follows:

$$
\begin{aligned}
& (0.851181615,1.983807346) \\
& (0.859282647,1.817826609) \\
& (0.995650019,2.570272620) \\
& (0.976747611,2.714122592)
\end{aligned}
$$

The four vertices of $D_{2}$ are:

(1.169822206, 3.798530079)

$(1.184674098,3.665745489)$

(1.257583385, 4.064099259)

(1.245431837, 4.196883850)

And the four vertices of $D_{3}$ are:

(1.404752132, 5.071049068)

$(1.416903680,4.938264478)$

(1.522217096, 5.535795134)

$(1.506015032,5.657514342)$

The quadrilaterals $D_{1}, D_{2}, D_{3}$ and their images under $H$ are given in Figure 13 .

We have the following proposition.

Proposition 3. When $a=3, c=6$ and $d=15$, the Poincaré map $H: P \rightarrow P$ has the property that there exists a closed invariant subset $\Lambda \subset P$, such that $H \mid \Lambda$ is 0.00,0.00,1.00 semi-conjugate to the 3-shift map. Hence, ent $(H) \geq \log 3>0$, which implies that system (3) has positive topological entropy with $a=3, c=6$ and $d=15$.

Proof. To prove this proposition, we only need to show the existence of a $H$-connected family $F$ with respect to $D_{1}, D_{2}$ and $D_{3}$.

As shown in Figure 13, $D_{1}^{1}, D_{2}^{1}$ and $D_{3}^{1}$ are the down side of $D_{1}, D_{2}$ and $D_{3}$, respectively, and $D_{1}^{2}, D_{2}^{2}$ and $D_{3}^{2}$ are the up side of $D_{1}, D_{2}$ and $D_{3}$, respectively. The image of $D_{1}, D_{2}$ and $D_{3}$ are $H\left(D_{1}\right), H\left(D_{2}\right)$ and $H\left(D_{3}\right)$, respectively.

As shown in Figure 13a, $H\left(D_{1}^{1}\right)$ lies below $D_{3}^{1}$, and $H\left(D_{1}^{2}\right)$ lies above $D_{2}^{2}, H\left(D_{1}\right)$ goes through $D_{i}$ with intersection points in $D_{i}{ }^{1}$ and $D_{i}{ }^{2}$ for each $i=1,2,3$. In this case, we say that the image $H\left(D_{1}\right)$ lies wholly across the quadrangles $D_{1}, D_{2}$ and $D_{3}$ with respect to $D_{3}^{1}$ and $D_{2}^{2}$.

Similarly, as shown in Figure 13b,c, $H\left(D_{2}^{1}\right)$ and $H\left(D_{3}^{1}\right)$ lie below $D_{3}^{1}$, and $H\left(D_{2}^{2}\right)$ and $H\left(D_{3}^{2}\right)$ lie above $D_{2}^{2}, H\left(D_{2}\right)$ and $H\left(D_{3}\right)$ go through $D_{i}$ with intersection points in $D_{i}{ }^{1}$ and $D_{i}{ }^{2}$ for each $i=1,2,3$. The images $H\left(D_{2}\right)$ and $H\left(D_{3}\right)$ lie wholly across the quadrangles $D_{1}, D_{2}$ and $D_{3}$ with respect to $D_{3}^{1}$ and $D_{2}^{2}$.

It is easy to see from Figure 13 that every line $\gamma$ connecting the side $D_{3}^{1}$ and $D_{2}^{2}$ has nonempty connection with $D_{1}, D_{2}$ and $D_{3}$. Further, based on the above arguments, $H\left(\gamma \cap D_{1}\right), H\left(\gamma \cap D_{1}\right)$ and $H\left(\gamma \cap D_{3}\right)$ connect $D_{3}^{1}$ and $D_{2}^{2}$. In view of Definition 1 , there exists a connection family with respect to 
these three subsets $D_{1}$ and $D_{2}$ for the map $H$. According to Theorem 1, there exists a compact invariant set $\Lambda \subset P$, such that $H \mid \Lambda$ is semi-conjugate to 3-shift map. It can be concluded from Theorem 2 that $\operatorname{ent}(H) \geq \operatorname{ent}(\sigma)=\log 3$, consequently, the entropy of the map $H$ is not less than $\log 3$, which implies system (3) has positive topological entropy with $a=3, c=6$ and $d=15$. Thus, this proof is completed.

To obtain the strict bounds of computer errors, we utilize the CAPD library, which is a package of flexible $\mathrm{C}++$ modules based on the Taylor method and the Lohner-type algorithm. The computations took a server (Xeon E5-2670) almost a week with MATLAB with MinGW-w64. The numerical errors for $H\left(D_{1}\right), H\left(D_{2}\right)$ and $H\left(D_{3}\right)$ are all less than $1 \times 10^{-5}$, which is too small to indicate in Figure 13.

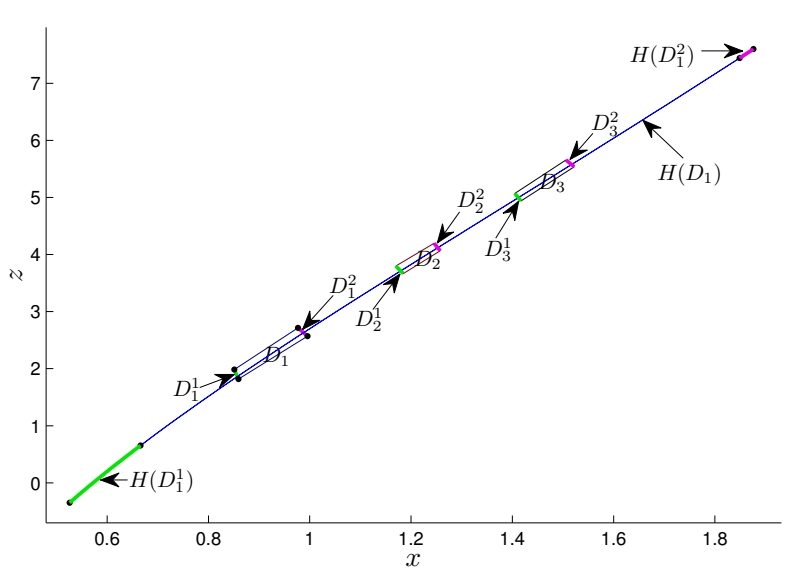

(a)

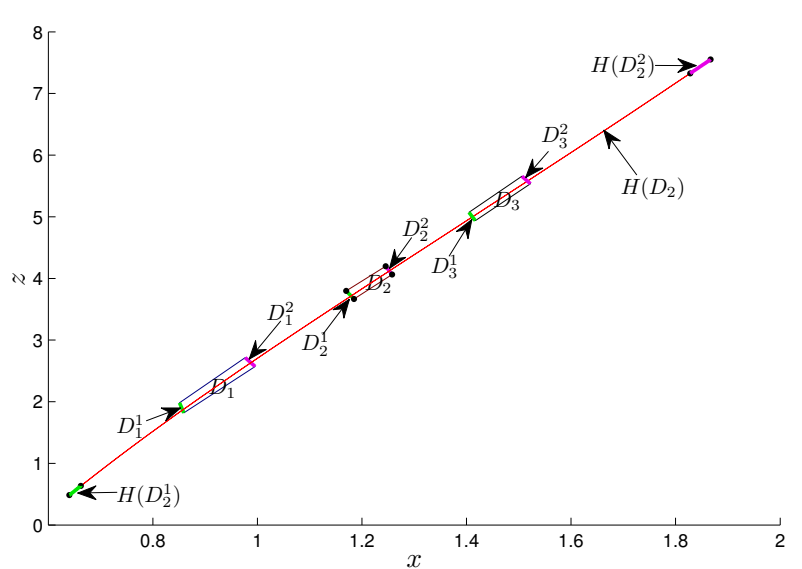

(b)

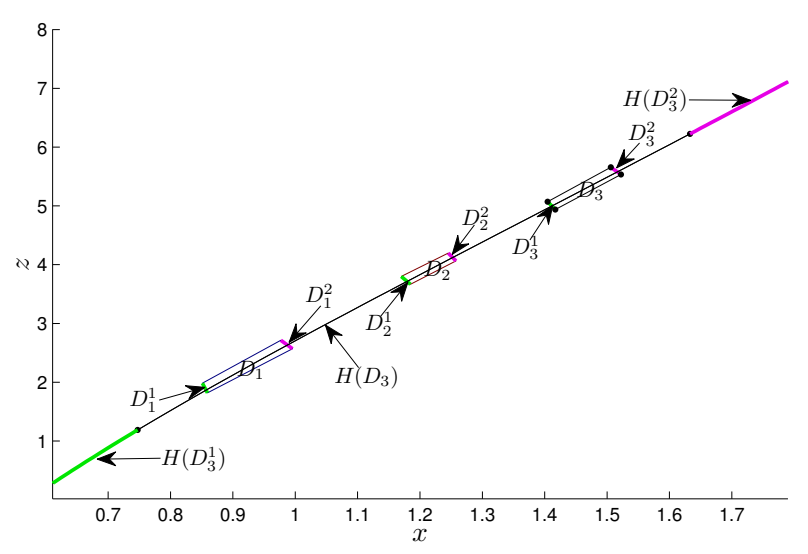

(c)

Figure 13. (a) $D_{1}, D_{2}, D_{3}$ and the image of $D_{1}$; (b) $D_{1}, D_{2}, D_{3}$ and the image of $D_{2}$; (c) $D_{1}, D_{2}, D_{3}$ and the image of $D_{3}$.

Since system (3) has positive topological entropy when $a=3, c=6$ and $d=15$, the attractor displayed in Figure 12 is chaotic indeed.

\section{Conclusions}

In this paper, we have presented and studied a new 3D quadratic autonomous system which can generate a butterfly chaotic attractor. By numerical simulations, we found that the Lyapunov dimension 
of the new system is greater than other known dissipative systems and the divergence of flow of this system is not a constant. The new double-scroll attractor could be split into the left or the right half-image attractors by adding a linear feedback control term into the second differential equation. Moreover, we proved that the Poincare map for the system is semi-conjugate to 3-shift map, which implies the topological entropy of this system is positive. In the future, we plan to focus on the mathematical analysis of the non-constant divergence.

\section{Acknowledgments}

We are grateful to the referees for their constructive comments and fruitful suggestions. This work is partially supported by Natural Science Foundation of China Grants No. 61174094, Tianjin Nature Science Foundation Grant No. 14JCYBJC18700, and Shandong Provincial Natural Science Foundation Grant No. ZR2014FQ019.

\section{Author Contributions}

During the development of this project, Zhonglin Wang and Zengqiang Chen conceived and designed the study, Jian Ma and Qing Zhang analyzed all figures and data for the paper, and Zhonglin Wang wrote the paper. All authors have read and approved the final manuscript.

\section{Conflicts of Interest}

The authors declare no conflict of interest.

\section{References}

1. Wang, S.; Kuang, J.; Li, J.; Luo, Y.; Lu, H.; Hu, G. Chaos-Based Secure Communications in a Large Community. Phys. Rev. E 2002, 66, 065202.

2. Zhang, W.; Wong, K.W.; Yu, H.; Zhu, Z.L. An Image Encryption Scheme Using Reverse 2-Dimensional Chaotic Map and Dependent Diffusion. Commun. Nonlinear Sci. Numer. Simul. 2013, 18, 147-148.

3. Yalcin, M.; Özoğuz, S.; Suykens, J. N-scroll Chaos Generators: A Simple Circuit Model. Electron. Lett. 2001, 37, 645-664.

4. Buscarino, A.; Fortuna, L.; Frasca, M.; Gambuzza, L.V. A Chaotic Circuit Based on Hewlett-Packard Memristor. Chaos 2012, 22, 023136.

5. Wang, J.; Tang, W.; Zhang, J. Approximate Synchronization of Two Non-linear Systems via Impulsive Control. Proc. Inst. Mech. Eng. Part I 2012, 226, 338-347.

6. Suykens, J.; Yalçın, M.; Vandewalle, J. Chaotic Systems Synchronization. In Chaos Control; Springer: Berlin, Germany, 2003; Volume 292, pp. 117-135.

7. Wu, X.; Lu, J.A.; Chi, K.T.; Wang, J.; Liu, J. Impulsive Control and Synchronization of the Lorenz Systems Family. Chaos Solitons Fractals 2007, 31, 631-638.

8. Lorenz, E.N. Deterministic Nonperiodic Flow. J. Atmos. Sci. 1963, 20, 130-141.

9. Rössler, O.E. An Equation for Continuous Chaos. Phys. Lett. A 1976, 57, 397-398. 
10. Chua, L.O.; Komuro, M.; Matsumoto, T. The Double Scroll Family. IEEE Trans. Circuits Syst. 1986, 33, 1073-1118.

11. Sprott, J. Some Simple Chaotic Flows. Phys. Rev. E 1994, 50, R647.

12. Chen, G.; Ueta, T. Yet Another Chaotic Attractor. Int. J. Bifurc. Chaos 1999, 9, 1465-1466.

13. May, R.M. Simple Mathematical Models with very Complicated Dynamics. Nature 1976, 261, 459-467.

14. Hénon, M. A Two-Dimensional Mapping with a Strange Attractor. Commun. Math. Phys. 1976, 50, 69-77.

15. Lozi, R. Un Attracteur Étrange (?) du Type Attracteur de Hénon. Le J. Phys. Colloq. 1978, 39 , C5-9-C5-10.

16. Lü, J.; Chen, G. A New Chaotic Attractor Coined. Int. J. Bifurc. Chaos 2002, 12, 659-661.

17. Smale, S. Differentiable Dynamical Systems. Bull. Am. Math. Soc. 1967, 73, 747-817.

18. Li, Q.; Yang, X.S. A Simple Method for Finding Topological Horseshoes. Int. J. Bifurc. Chaos 2010, 20, 467-478.

19. Li, Q.; Zhang, L.; Yang, F. An Algorithm to Automatically Detect the Smale Horseshoes. Discret. Dyn. Nat. Soc. 2012, 31, 726-737.

20. Zgliczynski, P. Computer Assisted Proof of Chaos in the Rössler Equations and in the Hénon Map. Nonlinearity 1997, 10, 243-252.

21. Mischaikow, K.; Mrozek, M. Chaos in the Lorenz Equations: A Computer-Assisted Proof. Bull. Am. Math. Soc. 1995, 32, 66-72.

22. Mischaikow, K.; Mrozek, M. Chaos in the Lorenz Equations: A Computer-Assisted Proof. Part II: Details. Math. Comput. Am. Math. Soc. 1997, 33, 66-72.

23. Li, Q.; Shu, C.; Ping, Z. Horseshoe and Entropy in a Fractional-Order Unified System. Chin. Phys. B 2011, 20, 010502.

24. Li, Q.; Yang, X.S. Chaotic Dynamics in a Class of Three Dimensional Glass Networks. Chaos Interdiscip. J. Nonlinear Sci. 2006, 16, 033101.

25. Huan, S.; Li, Q.; Yang, X.S. Horseshoes in a Chaotic System with only One Stable Equilibrium. Int. J. Bifurc. Chaos 2013, 01, 23.

26. Čelikovský, S.; Chen, G. On a Generalized Lorenz Canonical Form of Chaotic Systems. Int. J. Bifurc. Chaos 2002, 12, 1789-1812.

27. Čelikovský, S.; Chen, G. On the Generalized Lorenz Canonical Form. Chaos Solitons Fractals 2005, 26, 1271-1276.

28. Yang, Q.; Chen, G.; Zhou, T. A Unified Lorenz-type System and Its Canonical Form. Int. J. Bifurc. Chaos 2006, 16, 2855-2871.

29. Yang, Q.; Zhang, K.; Chen, G. A Modified Generalized Lorenz-type System and Its Canonical Form. Int. J. Bifurc. Chaos 2009, 19, 1931-1949.

30. Šil'nikov, L. A Contribution to the Problem of the Structure of an Extended Neighborhood of a Rough Equilibrium State of Saddle-Focus Type. Math. USSR SB 1970, 10, 91-102.

31. Shilnikov, L.P.; Shilnikov, A.L.; Turaev, D.V.; Chua, L.O. Methods of Qualitative Theory in Nonlinear Dynamics; World Scientific: Singapore, Singapore, 2001; pp. 393-957.

32. Sprott, J. Maximally Complex Simple Attractors. Chaos 2007, 17, 033124. 
33. Lü, J.; Chen, G.; Zhang, S. The Compound Structure of a New Chaotic Attractor. Chaos Solitons Fractals 2002, 14, 669-672.

34. Galias, Z. Positive Topological Entropy of Chua's Circuit: A Computer Assisted Proof. Int. J. Bifurc. Chaos 1997, 7, 331-349.

35. Galias, Z.; Zgliczyński, P. Computer Assisted Proof of Chaos in the Lorenz Equations. Physica D 1998, 115, 165-188.

36. Wiggins, S.; Golubitsky, M. Introduction to Applied Nonlinear Dynamical Systems and Chaos; Springer: Berlin, Germany, 1990; pp. 555-584.

37. Robinson, C. Dynamical Systems: Stability, Symbolic Dynamics, and Chaos; CRC Press: Boca Raton, FL, USA, 1995.

38. Yang, X.S.; Li, Q. A Computer-Assisted Proof of Chaos in Josephson Junctions. Chaos Solitons Fractals 2006, 27, 25-30.

39. Yang, X.S.; Tang, Y. Horseshoes in Piecewise Continuous Maps. Chaos Solitons Fractals 2004, $19,841-845$.

(c) 2015 by the authors; licensee MDPI, Basel, Switzerland. This article is an open access article distributed under the terms and conditions of the Creative Commons Attribution license (http://creativecommons.org/licenses/by/4.0/). 